Article

\title{
Multi-Attribute Decision Making Based on Probabilistic Neutrosophic Hesitant Fuzzy Choquet Aggregation Operators
}

\author{
Songtao Shao ${ }^{1,+}\left(\mathbb{D}\right.$, Xiaohong Zhang ${ }^{2,3, *} \mathbb{C}$ and Quan Zhao ${ }^{1,+}$ \\ 1 College of Information Engineering, Shanghai Maritime University, Shanghai 201306, China; \\ 201740310005@stu.shmtu.edu.cn (S.S.); quanzhao@shmtu.edu.cn (Q.Z.) \\ 2 Department of Mathematics, School of Arts and Sciences, Shaanxi University of Science \& Technology, \\ Xi'an 710021, China \\ 3 Department of Mathematics, College of Arts and Sciences, Shanghai Maritime University, \\ Shanghai 201306, China \\ * Correspondence: zhangxiaohong@sust.edu.cn or zhangxh@shmtu.edu.cn \\ + Current address: 1550, Haigang Main Road, Shanghai 201306, China.
}

Received: 21 March 2019; Accepted: 10 April 2019; Published: 3 May 2019

\begin{abstract}
Take the third-party logistics providers (3PLs) as an example, according to the characteristics of correlation between attributes in multi-attribute decision-making, two Choquet aggregation operators adoping probabilistic neutrosophic hesitation fuzzy elements (PNHFEs) are proposed to cope with the situations of correlation among criterions. This measure not only provides support for the correlation phenomenon between internal attributes, but also fully concerns the incidental uncertainty of the external space. Our goal is to make it easier for decision makers to cope with this uncertainty, thus we establish the notion of probabilistic neutrosophic hesitant fuzzy Choquet averaging (geometric) (PNHFCOA, PNHFCOG) operator. Based on this foundation, a method for aggregating decision makers' information is proposed, and then the optimal decision scheme is obtained. Finally, an example of selecting optimal 3PL is given to demonstrate the objectivity of the above-mentioned standpoint.
\end{abstract}

Keywords: probabilistic neutrosophic hesitant fuzzy set (PNHFS); decision-making; Choquet integral

\section{Introduction}

In the process of enterprise development, business leaders often encounter various multi-criteria decision-making (MADM) situations. In order for a company to survive in today's increasingly competitive real life, decision makers (DMs) must decide on the best solution when encountering MADM issues [1,2]. Therefore, how to effectively make optimal decisions in the MADM problems has become an emergency problem that global enterprises urgently need to solve. Establishing and perfecting research methods to suitable for MADM situations has attracted more and more attention from DMs [3]. The key to MADM is to choose the appropriate decision theory and computer software to aggregate the information of DMs and make the best decision in the decision process according to relevance of the information. In order to choose the optimal solution, different MADM schemes have been established to increase the development and competitiveness of enterprises. Since the most common feature in decision information is the ambiguity of the information. Therefore, some related researches based on fuzzy information have been proposed [4-12].

In reality, there is a correlation among attributes in the process of MADM. In addition, some researchers have begun to pay attention to this issue. For example, Brito et al. [13] proposed a new-type multi-criteria model based on the Choquet integral and epistemic mapping technique 
for evaluating eco-friendly cities. Krishnan et al. [14] introduced a new $\lambda^{0}$-measure authentication approach that expresses the mutual relation between the attributes. To more effectively highlight the correlation between attributes, Beg et al. [15] introduced the diminishing Chouqet hesitant 2-tuple average (DCH2TA) operator and established a MADM approach.

Compared with reality, the current research method does not consider the fuzzy information with possibility in the MADM problem. Thus, when there is a situation with possibility information, the existing methods will be invalid. To deal with this type of problem, there are two aspects that need to be solved: one is the representation of the PNHF information and the probability information of external environment. The other is the establishment of the MADM model considering the interrelationship among the attributes.

According to the analysis of the common fuzzy conditions in practice, fuzzy sets [16] have been used in many situations. Diversity based on fuzzy information, the fuzzy set theory has been continuously improved. For example, intuitionistic FS [17], hesitant FS [18], and dual hesitant FS [19]. When dealing with fuzzy data, DMs will often encounter the following three kinds of independent fuzzy information: truth fuzzy information, indeterminacy fuzzy information and falsity fuzzy information. The definition of neutrosophic set (NS) was constructed [20] to express the feature. For application to engineering projects, different types of generalized NSs are constructed such as single-valued NS [21], interval NS [22], and neutrosophic hesitant fuzzy (NHF) set [23]. These extended NS theories have been applied to medical diagnosis and other engineering fields [24-28]. Fuzzy set and neutrosophic set are also extended to the field of algebra [29-33]. In order to express three independent hesitant messages, NHFS was proposed and applied to MADM [34-36]. However, as can be seen from these references, those theories can only express information about cognitive uncertainty. Thus, in this article, we use PNHFS [3,37] to express the occasional uncertainty of information and the uncertainty of cognition. Under the MADM environment, due to the different cognitive situations of DMs and their own hesitation, the final evaluation value may not be unique. Depending on the complexity of the external environment, the probability of taking values also affects the evaluation values. Thus, probability plays a key role in interpreting the evaluation value, which avoid the loss of evaluation values, simultaneously. Not only subjective information of NHFS, but also objective probability information of each membership was clearly described.

The MADM problem under attribute correlation is one of the main research questions in this paper. As an important part of fuzzy mathematics, fuzzy integral can help DMs to better deal with MADM problems through modeling methods when attributes are associated with each other. The classic weight information is to satisfy the additivity condition, and the advantage of fuzzy integral is that it is not limited by the additivity condition, that is, the sum of the fuzzy integral may be greater than 1. When the Choquet integral (CI) [38] satisfies the additivity, the Choquet integral is converted into classic weight information. Therefore, the application range of Choquet integral is extensive, and it is more suitable for coping with MADM problems with uncertain information. For example, Khan et al. [39] proposed the (generalized) Pythagorean hesitant fuzzy Choquet averaging (geometric) operators under the MADM environment. Based on the hierarchical and interacting standards, Corrente et al. [40] Choquet integral preference model that can be robust ranking of universities evaluation. Labreuche et al. [41] developed two new Choquet models. Yager [42] used the standard Shapley value as an approximation of Choquet integra. Liu et al. [43] effectively investigate the MADM situations that the interrelationship between attributes, globally. Absolutely, above aggregation operators can not integrate PNHF information. Thus, we construct a new integration method by CI. Thus, we extend these operators to the PNHFSs and propose PNHF Choquet averaging (geometric) operators and establish a process to handle MADM situations.

Based on the above analysis: In Section 2, fundamental concepts are reviewed. In Section 3, the operators are included, the comparison approaches are described, and some basic properties are studied. In Section 4, an approach to MADM based on the PNHFCOA (PNHFCOG) operator is proposed. In Section 5, an illustrative situation is given to confirm the proposed method. In Section 6, our results are analyzed with the results of other methods. Finally, conclusions and future work are summarized. 


\section{Preliminaries}

Some basic definitions can be referred to $[3,23,37,38,44]$.

Definition 1. A NHF set (NHFS) in a reference domain X set is depicted by:

$$
N=\{\langle x, \tilde{t}(x), \tilde{i}(x), \tilde{f}(x)\rangle \mid x \in X\}
$$

in which $\tilde{t}(x)$ indicates that a set includes some truth-membership hesitant degrees of $x, \tilde{i}(x)$ indicates that a set includes some indeterminacy-membership hesitant degrees, and $\tilde{f}(x)$ indicates that a set includes some falsity-membership hesitant degrees. The following conditions are held: $\delta \in \tilde{t}(x), \gamma \in \tilde{i}(x), \eta \in \tilde{f}(x)$, $\delta, \gamma, \eta \in[0,1], \max \{\delta\}+\max \{\gamma\}+\max \{\eta\} \in[0,3]$.

Definition 2. A PNHFS in a reference domain $X$ is depicted by:

$$
N=\left\{\left\langle x, T(x)\left|P^{T}(x), I(x)\right| P^{I}(x), F(x) \mid P^{F}(x)\right\rangle \mid x \in X\right\} .
$$

$T(x)\left|P^{T}(x), I(x)\right| P^{I}(x)$ and $F(x) \mid P^{F}(x)$ describes three components of $N, T(x), I(x)$ and $F(x)$ is depicted the three types hesitant degrees of $x . P^{T}(x), P^{I}(x)$ and $P^{F}(x)$ describe the corresponding probability of $T(x)$, $I(x)$ and $F(x)$, The conditions that need to be held:

$$
\begin{gathered}
\alpha \in T(x), \beta \in I(x), \gamma \in F(x), \alpha, \beta, \gamma \in[0,1], \max \{\alpha\}+\max \{\beta\}+\max \{\gamma\} \in[0,3] \\
P_{a}^{T} \in P^{T}, P_{b}^{I} \in P^{I}, P_{c}^{F} \in P^{F} . P_{a}^{T}, P_{b}^{I}, P_{c}^{F} \in[0,1] ; \sum_{a=1}^{\# \tilde{t}} P_{a}^{T} \leq 1, \sum_{b=1}^{\# \tilde{i}} P_{b}^{I} \leq 1, \sum_{c=1}^{\# \tilde{f}} P_{c}^{F} \leq 1 .
\end{gathered}
$$

$\# \tilde{t}, \# \tilde{i}$ and \# $\tilde{f}$ describe the cardinal number of $T(x)\left|P^{T}(x), I(x)\right| P^{I}(x), F(x) \mid P^{F}(x)$, respectively.

Generally, $N=\left\{T\left|P^{T}, I\right| P^{I}, F \mid P^{F}\right\}$ is described $a$ an PNHF number (PNHFE) of $N=$ $\left\langle T(x)\left|P^{T}(x), I(x)\right| P^{I}(x), F(x) \mid P^{F}(x)\right\rangle$.

Definition 3. A normalized PNHFE N satisfies the following conditions:

$$
\tilde{N}=\left\langle T(x)\left|\tilde{P}^{T}(x), I(x)\right| \tilde{P}^{I}(x), F(x) \mid \tilde{P}^{F}(x)\right\rangle,
$$

where $\tilde{P}_{a}^{T}=\frac{P_{a}^{T}}{\sum P_{a}^{T}}, \tilde{P}_{b}^{I}=\frac{P_{b}^{I}}{\sum P_{b}^{I}}, \tilde{P}_{c}^{F}=\frac{P_{c}^{F}}{\sum P_{c}^{F}}$

Definition 4. Supposing that $N_{1}=\left\{T_{1}\left|P^{T_{1}}, I_{1}\right| P^{I_{1}}, F_{1} \mid P^{F_{1}}\right\}, N_{2}=\left\{T_{2}\left|P^{T_{2}}, I_{2}\right| P^{I_{2}}, F_{2} \mid P^{F_{2}}\right\}$ are two PNHFEs, some basic algorithms are defined by:

(1) $\left(N_{1}\right)^{c}=\bigcup_{\alpha_{1} \in T_{1}, \beta_{1} \in I_{1}, \gamma_{1} \in F_{1}}\left\{\gamma_{1}\left|P_{1}^{F_{1}}, 1-\beta_{1}\right| P_{1}^{I_{1}}, \alpha_{1} \mid P_{1}^{T_{1}}\right\}$,

(2) $\left(N_{1}\right)^{\lambda}=\bigcup_{\alpha_{1} \in T_{1}, \beta_{1} \in I_{1}, \gamma_{1} \in F_{1}}\left\{\left\{\left(\alpha_{1}\right)^{\lambda} \mid P_{1}^{T_{1}}\right\},\left\{1-\left(1-\beta_{1}\right)^{\lambda} \mid P_{1}^{I_{1}}\right\},\left\{1-\left(1-\gamma_{1}\right)^{\lambda} \mid P_{1}^{F_{1}}\right\}\right\}$,

(3) $\lambda\left(N_{1}\right)=\underset{\alpha_{1} \in T_{1}, \beta_{1} \in I_{1}, \gamma_{1} \in F_{1}}{ }\left\{\left\{1-\left(1-\lambda_{1}\right)^{\lambda} \mid P_{1}^{T_{1}}\right\},\left\{\left(\beta_{1}\right)^{\lambda} \mid P_{1}^{I_{1}}\right\},\left\{\left(\gamma_{1}\right)^{\lambda} \mid P_{1}^{F_{1}}\right\}\right\}$,

(4) $N_{1} \oplus N_{2}=\bigcup_{\substack{\alpha_{1} \in T_{1}, \beta_{1} \in I_{1}, \gamma_{1} \in F_{1}, \eta_{2} \in T_{2}, \pi_{2} \in I_{2}, \mu_{2} \in F_{2}}}\left\{\left\{\alpha_{1}+\eta_{2}-\alpha_{2} \eta_{2} \mid P_{1}^{T_{1}} P_{2}^{T_{2}}\right\},\left\{\beta_{1} \pi_{2} \mid P_{1}^{I_{1}} P_{2}^{I_{2}}\right\},\left\{\gamma_{1} \mu_{2} \mid P_{1}^{F_{1}} P_{2}^{F_{2}}\right\}\right\}$,

(5) $N_{1} \otimes N_{2}=\bigcup_{\substack{\alpha_{1} \in T_{1}, \beta_{1} \in I_{1}, \gamma_{1} \in F_{1}, \eta_{2} \in T_{2}, \pi_{2} \in I_{2}, \mu_{2} \in F_{2}}}\left\{\left\{\alpha_{1} \eta_{2} \mid P_{1}^{T_{1}} P_{2}^{T_{2}}\right\},\left\{\beta_{1}+\pi_{2}-\beta_{1} \pi_{2} \mid P_{1}^{I_{1}} P_{2}^{I_{2}}\right\},\left\{\gamma_{1}+\mu_{2}-\gamma_{1} \mu_{2} \mid P_{1}^{F_{1}} P_{2}^{F_{2}}\right\}\right\}$,

where $P_{1}^{T_{1}} ; P_{1}^{I_{1}}$ and $P_{1}^{F_{1}}$ are hesitant probabilities of $\alpha_{1} \in T_{1}, \beta_{1} \in I_{1}$ and $\gamma_{1} \in F_{1}$, respectively. $P_{2}^{T_{2}} ; P_{2}^{I_{2}}$ and $P_{1}^{F_{2}}$ are corresponding hesitant probabilities of $\eta_{2} \in T_{2}, \pi_{2} \in I_{2}$ and $\mu_{2} \in F_{2}$. 
Definition 5. $P(Y)$ depicts the power set of a domain $Y$. Fuzzy measure $\mu: P(Y) \rightarrow[0,1]$ satisfies conditions:

(1) $\mu(\varnothing)=0, \mu(Y)=1$;

(2) $A \subseteq B$, then $\mu(A) \leq \mu(B), \forall A, B \subseteq P(Y)$;

Fuzzy measure $\mu$ satisfies property, $\forall Y_{1}, Y_{2} \in P(Y), A \cap B=\varnothing$

$$
\mu\left(Y_{1} \cup Y_{2}\right)=\mu\left(Y_{1}\right)+\mu\left(Y_{2}\right)+\lambda \mu\left(Y_{1}\right) \mu\left(Y_{2}\right), \lambda \in(-1, \infty) .
$$

Then, $\mu$ is described a $\lambda$-fuzzy measure.

Theorem 1. A $\lambda$-fuzzy measure $\mu$ in a discourse $Y=\left\{y_{1}, y_{2}, \cdots, y_{n}\right\}$ satisfies the following formula:

$$
\mu(X)= \begin{cases}\frac{1}{\lambda}\left(\prod_{i=1}^{n}\left[1+\lambda \mu\left(y_{i}\right)\right]-1\right) & \text { if } \lambda \neq 0, \\ \sum_{i=1}^{n} \mu\left(y_{i}\right) & \text { if } \lambda=0 .\end{cases}
$$

Since $\mu(Y)=1$, parameter $\lambda$ can be determined by

$$
\lambda=\prod_{i=1}^{n}\left(1+\lambda \mu\left(x_{i}\right)\right)-1 .
$$

Definition 6. $f$ is a real function on $Y=\left\{y_{1}, y_{2}, \cdots, y_{n}\right\}$. The Choquet integral about fuzzy measure $\mu$ is depicted by:

$$
\int f d \mu=\sum_{a=1}^{n} f\left(y_{(a)}\right)\left[\mu\left(A_{(a)}\right)-\mu\left(A_{(a+1)}\right)\right]
$$

in which $\left\{x_{\pi(1)}, x_{\pi(2)}, \cdots, x_{\pi(n)}\right\}$ is a new rank of $Y, f\left(y_{\pi(1)}\right) \leq f\left(y_{\pi(2)}\right) \leq \cdots \leq f\left(y_{\pi(n)}\right)$, $A_{(a)}=\left\{y_{\pi(a)}, y_{\pi(a+1)}, \cdots, x_{\pi(n)}\right\}$ and $A_{(n+1)}=0$.

\section{PNHFSs and Aggregation Operators}

The PNHFCOA and PNHFCOG operators are proposed in this section. Some basic properties are verified.

\subsection{The Comparison Method of PNHFEs}

When we describe decision information with PNHFS theory, a comparison method of PNHFEs is necessary. Thus, a approch of ranking PNHFEs is established.

Definition 7. Supposing that $N=\left\{T\left|P^{T}, I\right| P^{I}, F \mid P^{F}\right\}$ is an PNHFE, then the score function of the PNHFE is expressed by the following formula:

$$
S(N)=\sum_{a=1}^{\# T} \alpha_{a} P_{a}^{T}+\sum_{b=1}^{\# I}\left(1-\beta_{b}\right) P_{b}^{I}-\sum_{c=1}^{\# F} \gamma_{c} P_{c}^{F} .
$$

Definition 8. Supposing that $N=\left\{T\left|P^{T}, I\right| P^{I}, F \mid P^{F}\right\}$ is an PNHFE, then we can find the deviation function $D(N)$ :

$$
D(N)=\sum_{a=1}^{\# T}\left(\alpha_{a}-S(N)\right)^{2} \cdot P_{a}^{T}+\sum_{b=1}^{\# I}\left(1-\beta_{b}-S(N)\right)^{2} \cdot P_{b}^{I}+\sum_{c=1}^{\# F}\left(\gamma_{c}-S(N)\right)^{2} \cdot P_{c}^{F} .
$$

The distance from the score valued in the PNHFE $N$ is described by the deviation function. Thus, the deviation value is called a consistency indicator of the PNHFE $N$. The higher value of $D(N)$, the lower consistency of $N$. Based on Definitions 7 and 8, a method for ranking two PNHFEs is developed. 
Definition 9. For PNHFEs $N_{1}$ and $N_{2}$, the sort of $N_{1}$ and $N_{2}$ is determined by:

(1) If $S\left(N_{1}\right)>S\left(N_{2}\right)$, it indicates that PNHFE $N_{1}$ is superior to $N_{2}$;

(2) If $S\left(N_{1}\right)=S\left(N_{2}\right), D\left(N_{1}\right)>D\left(N_{2}\right)$, it indicates that PNHFE $N_{1}$ is inferior to $N_{2}$;

(3) If $S\left(N_{1}\right)=S\left(N_{2}\right), D\left(N_{1}\right)=D\left(N_{2}\right)$, it indicates that PNHFE $N_{1}$ is equal to $N_{2}$.

\subsection{The PNHFCOA Operator and PNHFCOG Operator}

This section constructed the PNHFCOA operator and PNHFCOG operator under the probabilistic neutrosophic environment, and some basic properties are investigated. In this section, $\mu$ describes a fuzzy measure on a domain $X, k=1,2, \cdots, n$.

Definition 10. Suppose that $N_{k}=\left\{T_{k}\left|P^{T_{k}}, I_{k}\right| P^{I_{k}}, F_{k} \mid P^{F_{k}}\right\}$ describes an PNHFE in a reference set $X$. Then, the PNHFCOA operator is expressed by the following formula:

$$
\begin{aligned}
& \operatorname{PNHFCOA}\left(N_{1}, N_{2}, \cdots, N_{n}\right)=\oplus_{\pi(k)=1}^{n} \mu_{\pi(k)} N_{\pi(k)} \\
& =\bigcup_{\alpha_{\pi(k)} \in T_{\pi(k)}, \beta_{\pi(k)} \in I_{\pi(k)}, \gamma_{\pi(k)} \in F_{\pi(k)}}^{n}\left\{\left\{1-\prod_{\pi(k)=1}^{n}\left(1-\alpha_{\pi(k)}\right)^{\mu_{\pi(k)}} \mid \prod_{\pi(k)=1}^{n} P_{\pi(k)}^{T_{\pi(k)}}\right\},\right. \\
& \left.\left\{\prod_{\pi(k)=1}^{n}\left(\beta_{\pi(k)}\right)^{\mu_{\pi(k)}} \mid \prod_{\pi(k)=1}^{n} P_{\pi(k)}^{I_{\pi(k)}}\right\},\left\{\prod_{\pi(k)=1}^{n}\left(\gamma_{\pi(k)}\right)^{\mu_{\pi(k)}} \mid \prod_{\pi(k)=1}^{n} P_{\pi(k)}^{F_{\pi}(k)}\right\}\right\},
\end{aligned}
$$

where $P_{\pi(k)}^{T_{\pi(k)}}, P_{\pi(k)}^{I_{\pi(k)}}$ and $P_{\pi(k)}^{F_{\pi(k)}}$ are corresponding probability data of $\alpha_{\pi(k)}, \beta_{\pi(k)}$ and $\gamma_{\pi(k)} \cdot \mu_{\pi(k)}=\mu\left(F_{\pi(k)}-\right.$ $\left.F_{\pi(i+1)}\right), F_{\pi(k)}=\left\{x_{\pi(1)}, x_{\pi(2)}, \cdots, x_{\pi(k)}\right\}$ and $F_{\pi(0)}=0 .\left\{n_{\pi(k)}\right\}$ is a sequence such that $n_{\pi(1)} \leq n_{\pi(2)} \leq$ $\cdots \geq n_{\pi(m)}$.

Theorem 2. Supposing that $N_{k}$ describes an PNHFE, PNHFCOA operator PNHFCOA $\left(N_{1}, N_{2}, \cdots, N_{n}\right)$ is still an PNHFE.

Proof. The mathematical induction can be utilized.

(1) When $n=1$, we have the following equation by Definition 10 :

$$
\operatorname{PNHFCOA}\left(N_{1}\right)=\mu_{\pi(1)} \oplus N_{\pi(1)}=N_{1}
$$

Obviously, PNHFCOA $\left\{N_{1}\right\}$ is an PNHFE.

(2) When $n=2$, we have

$$
\begin{aligned}
& \operatorname{PNHFCOA}\left(N_{1}, N_{2}\right)=\left(\mu_{\pi(1)} N_{\pi(1)}\right) \oplus\left(\mu_{\pi(2)} N_{\pi(2)}\right) \\
& =\bigcup_{\alpha_{\pi(1)} \in T_{\pi(1)}, \beta_{\pi(1)} \in I_{\pi(1)}, \gamma_{\pi(1)} \in F_{\pi(1)}}\left\{1-\left(1-\alpha_{\pi(1)}\right)^{\mu_{\pi(1)}}\left|P_{\pi(1)}^{T_{\pi(1)}}, \beta_{\pi(1)}^{\mu_{\pi(1)}}\right| P_{\pi(1)}^{I_{\pi(1)}}, \gamma_{\pi(1)}^{\mu_{\pi(1)}} \mid P_{\pi(1)}^{F_{\pi(1)}}\right\} \\
& \bigcup_{\alpha_{\pi(2)} \in T_{\pi(2)}, \beta_{\pi(2)} \in I_{\pi(2)}, \gamma_{\pi(2)} \in F_{\pi(2)}}\left\{1-\left(1-\alpha_{\pi(2)}\right)^{\mu_{\pi(2)} \mid}\left|P_{\pi(2)}^{T_{\pi(2)}}, \beta_{\pi(2)}^{\mu_{\pi(2)}}\right| P_{\pi(2)}^{I_{\pi(2)}}, \gamma_{\pi(2)}^{\mu_{\pi(2)}} \mid P_{\pi(2)}^{F_{\pi(2)}}\right\} \\
& =\bigcup_{\alpha_{\pi(k)} \in T_{\pi(k)}, \beta_{\pi(k)} \in I_{\pi(k)}, \gamma_{\pi(k)} \in F_{\pi(k)}}^{2}\left\{1-\prod_{\pi(k)=1}^{2}\left(1-\alpha_{\pi(k)}\right)^{\mu_{\pi(k)}} \mid \prod_{\pi(k)=1}^{2} P_{\pi(1)}^{T_{\pi(k)},}\right. \\
& \left.\prod_{\pi(k)=1}^{2} \beta_{\pi(k)}^{\mu_{\pi(k)} \mid} \prod_{\pi(k)=1}^{2} P_{\pi(k)}^{I_{\pi(k)}} \prod_{\pi(k)=1}^{2} \gamma_{\pi(k)}^{\mu_{\pi(k)}} \mid \prod_{\pi(k)=1}^{2} P_{\pi(k)}^{F_{\pi(k)}}\right\} .
\end{aligned}
$$

Thus, we know PNHFCOA $\left\{N_{1}, N_{2}\right\}$ is an PNHFE.

(3) When $n=k$, Equation (9) is true, and we have 


$$
\begin{aligned}
& \operatorname{PNHFCOA}\left(N_{1}, N_{2}, \cdots, N_{k}\right)=\oplus_{\pi(k)=1}^{k} \mu_{\pi(k)} N_{\pi(k)} \\
& =\bigcup_{\alpha_{\pi(k)} \in T_{\pi(k)}, \beta_{\pi(k)} \in I_{\pi(k)}, \gamma_{\pi(k)} \in F_{\pi(k)}}\left\{1-\prod_{\pi(k)=1}^{k}\left(1-\alpha_{\pi(k)}\right)^{\mu_{\pi(k)}} \mid \prod_{\pi(k)=1}^{k} P_{\pi(k)}^{T_{\pi(k)}}\right\}, \\
& \left.\left\{\prod_{\pi(k)=1}^{k}\left(\beta_{\pi(k)}\right)^{\mu_{\pi(k)}} \mid \prod_{\pi(k)=1}^{k} P_{\pi(k)}^{I_{\pi(k)}}\right\},\left\{\prod_{\pi(k)=1}^{k}\left(\gamma_{\pi(k)}\right)^{\mu_{\pi(k)}} \mid \prod_{\pi(k)=1}^{k} P_{\pi(k)}^{F_{\pi}(k)}\right\}\right\} .
\end{aligned}
$$

Thus, the next formula is obtained, $n=k+1$,

$$
\begin{aligned}
& \operatorname{PNHFCOA}\left(N_{1}, N_{2}, \cdots, N_{k}, N_{k+1}\right)=\left(\oplus_{\pi(k)=1}^{k} \mu_{\pi(k)} N_{\pi(k)}\right) \oplus\left(\mu_{\pi(k+1)} N_{\pi(k+1)}\right) \\
& =\bigcup_{\alpha_{\pi(k)} \in T_{\pi(k)}, \beta_{\pi(k)} \in I_{\pi(k)}, \gamma_{\pi(k)} \in F_{\pi(k)}}\left\{\left\{1-\prod_{\pi(k)=1}^{k}\left(1-\alpha_{\pi(k)}\right)^{\mu_{\pi(k)}} \mid \prod_{\pi(k)=1}^{k} P_{\pi(k)}^{T_{\pi(k)}}\right\},\right. \\
& \left.\left\{\prod_{\pi(k)=1}^{k}\left(\beta_{\pi(k)}\right)^{\mu_{\pi(k)}} \mid \prod_{\pi(k)=1}^{k} P_{\pi(k)}^{I_{\pi(k)}}\right\},\left\{\prod_{\pi(k)=1}^{k}\left(\gamma_{\pi(k)}\right)^{\mu_{\pi(k)}} \mid \prod_{\pi(k)=1}^{k} P_{\pi(k)}^{F_{\pi}(k)}\right\}\right\} \\
& \oplus \bigcup_{\alpha_{\pi(k+1)} \in T_{\pi(k+1)}, \beta_{\pi(k+1)} \in I_{\pi(k+1)}, \gamma_{\pi(k+1)} \in F_{\pi(k+1)}}\left\{1-\left(1-\alpha_{\pi(k+1)}\right)^{\mu_{\pi(k+1)}} \mid P_{\pi(k+1)}^{T_{\pi(k+1)}}\right. \\
& \left.\beta_{\pi(k+1)}^{\mu_{\pi(k+1)}}\left|P_{\pi(k+1)}^{I_{\pi(k+1)}}, \gamma_{\pi(k+1)}^{\mu_{\pi(k+1)}}\right| P_{\pi(k+1)}^{F_{\pi(k+1)}}\right\} \\
& =\bigcup_{\alpha_{\pi(k)} \in T_{\pi(k)}, \beta_{\pi(k)} \in I_{\pi(k)}, \gamma_{\pi(k)} \in F_{\pi(k)}}\left\{\left\{1-\prod_{\pi(k)=1}^{k+1}\left(1-\alpha_{\pi(k)}\right)^{\mu_{\pi(k)}} \mid \prod_{\pi(k)=1}^{k+1} P_{\pi(k)}^{T_{\pi(k)}}\right\},\right. \\
& \left.\left\{\prod_{\pi(k)=1}^{k+1}\left(\beta_{\pi(k)}\right)^{\mu_{\pi(k)}} \mid \prod_{\pi(k)=1}^{k+1} P_{\pi(k)}^{I_{\pi(k)}}\right\},\left\{\prod_{\pi(k)=1}^{k+1}\left(\gamma_{\pi(k)}\right)^{\mu_{\pi(k)}} \mid \prod_{\pi(k)=1}^{k+1} P_{\pi(k)}^{F_{\pi}(k)}\right\}\right\} .
\end{aligned}
$$

Thus, for any $n$, the conclusion is right.

Next, when the fuzzy measure satisfies different conditions, different types of PNHFCOA can be obtained.

(1) Assume $\mu(F)=1$, then

$$
\operatorname{PNHFCOA}\left(N_{1}, N_{2}, \cdots, N_{n}\right)=\max \left\{N_{1}, N_{2}, \cdots, N_{n}\right\} .
$$

(2) Assume $\mu(F)=0$, then

$$
\operatorname{PNHFCOA}\left(N_{1}, N_{2}, \cdots, N_{n}\right)=\min \left\{N_{1}, N_{2}, \cdots, N_{n}\right\} .
$$

(3) Assume the condition $\mu\left(x_{\pi(k)}\right)=\mu\left(F_{\pi(k)}-F_{\pi(k-1)}\right)$ is independent, the PNHFCOA operator is described an PNHFWA operator,

$$
\begin{aligned}
& \operatorname{PNHFWA}\left(N_{1}, N_{2}, \cdots, N_{n}\right)=\oplus_{k=1}^{n} \mu\left(x_{k}\right) N_{k} \\
& =\bigcup_{\alpha_{k} \in T_{k}, \beta_{k} \in I_{k}, \gamma_{k} \in F_{k}}\left\{\left\{1-\prod_{k=1}^{n}\left(1-\alpha_{k}\right)^{\mu\left(x_{k}\right)} \mid \prod_{k=1}^{n} P_{k}^{T_{k}}\right\},\left\{\prod_{k=1}^{n}\left(\beta_{k}\right)^{\mu\left(x_{k}\right)} \mid \prod_{k=1}^{n} P_{k}^{I_{k}}\right\},\left\{\prod_{k=1}^{n}\left(\gamma_{k}\right)^{\mu\left(x_{k}\right)} \mid \prod_{k=1}^{n} P_{k}^{F_{k}}\right\}\right\} .
\end{aligned}
$$

(4) Assume the condition $\mu\left(x_{\pi(k)}\right)=\frac{1}{n}$, the PNHFCOA operator and PNHFWA operator reduce to the PNHFA operator, 


$$
\begin{aligned}
& \operatorname{PNHFWA}\left(N_{1}, N_{2}, \cdots, N_{n}\right)=\oplus_{k=1}^{n} \mu\left(x_{k}\right) N_{k} \\
& =\bigcup_{\alpha_{k} \in T_{k}, \beta_{k} \in I_{k}, \gamma_{k} \in F_{k}}\left\{\left\{1-\prod_{k=1}^{n}\left(1-\alpha_{k}\right)^{\frac{1}{n}} \mid \prod_{k=1}^{n} P_{k}^{T_{k}}\right\},\left\{\prod_{k=1}^{n}\left(\beta_{k}\right)^{\frac{1}{n}} \mid \prod_{k=1}^{n} P_{k}^{I_{k}}\right\},\left\{\prod_{k=1}^{n}\left(\gamma_{k}\right)^{\frac{1}{n}} \mid \prod_{k=1}^{n} P_{k}^{F_{k}}\right\}\right\} .
\end{aligned}
$$

Theorem 3. (Monotonicity) Suppose $N_{k}=\left\{\left\{\alpha_{k} \mid P_{k}^{T_{k}}\right\},\left\{\beta_{k} \mid P_{k}^{I_{k}}\right\},\left\{\gamma_{k} \mid P_{k}^{F_{k}}\right\}\right\}$ and $\tilde{N}_{k}=\left\{\left\{\tilde{\alpha}_{k} \mid P_{k}^{\tilde{T}_{k}}\right\},\left\{\tilde{\beta}_{k} \mid P_{k}^{\tilde{I}_{k}}\right\}\right.$, $\left.\left\{\tilde{\gamma}_{k} \mid P_{k}^{\tilde{F}_{k}}\right\}\right\}$ indicate two PNHFEs. The factor $\pi(k)$ satisfies $N_{\pi(1)} \geq N_{\pi(2)} \geq \cdots \geq N_{\pi(n)}$ and $\tilde{N}_{\pi(1)} \geq$ $\tilde{N}_{\pi(2)} \geq \cdots \geq \tilde{N}_{\pi(n)}$. For any $N_{\pi(k)}, \tilde{N}_{\pi(k)}$, there are $\alpha_{\pi(k)} \leq \tilde{\alpha}_{\pi(k)}, \beta_{\pi(k)} \geq \tilde{\beta}_{\pi(k)}, \gamma_{\pi(k)} \geq \tilde{\gamma}_{\pi(k)}$ and $P_{\pi(k)}^{T_{\pi(k)}}=P_{\pi(k)}^{\tilde{T}_{\pi(k)}}, P_{\pi(k)}^{I_{\pi(k)}}=P_{\pi(k)}^{\tilde{I}_{\pi(k)}}, P_{\pi(k)}^{F_{\pi(k)}}=P_{\pi(k)}^{\tilde{F}_{\pi(k)}}$. Then,

$$
\operatorname{PNHFCOA}\left\{N_{1}, N_{2}, \cdots, N_{n}\right\} \leq \operatorname{PNHFCOA}\left\{\tilde{N}_{1}, \tilde{N}_{2}, \cdots, \tilde{N}_{n}\right\} .
$$

Proof. By Definition $7, N_{\pi(k)} \leq \tilde{N}_{\pi(k)}$. By Definition 10, the following inequality is obtained:

$$
\begin{gathered}
\left.\left(1-\Pi\left(1-\alpha_{\pi(k)}\right)^{\mu_{\pi(k)}}\right) \prod P_{\pi(k)}^{T_{\pi(k)}}+\left(1-\Pi\left(\beta_{\pi(k)}\right)^{\mu_{\pi(k)}} \prod P_{\pi(k)}^{I_{\pi(k)}}\right)-\Pi\left(\gamma_{\pi(k)}\right)^{\mu_{\pi(k)}} \Pi P_{j}^{F_{\pi(k)}}\right\} \leq \\
\left.\left(1-\Pi\left(1-\tilde{\alpha}_{\pi(k)}\right)^{\mu_{\pi(k)}}\right) \prod P_{\pi(k)}^{T_{\pi(k)}}+\left(1-\Pi\left(\tilde{\beta}_{\pi(k)}\right)^{\mu_{\pi(k)}} \prod P_{\pi(k)}^{I_{\pi(k)}}\right)-\Pi\left(\tilde{\gamma}_{\pi(k)}\right)^{\mu_{\pi(k)}} \prod P_{j}^{F_{\pi(k)}}\right\} .
\end{gathered}
$$

Then, by Definitions 7 and 9 , the result is proved

$$
\operatorname{PNHFCOA}\left(N_{1}, N_{2}, \cdots, N_{n}\right) \leq \operatorname{PNHFCOA}\left(\tilde{N}_{1}, \tilde{N}_{2}, \cdots, \tilde{N}_{n}\right) .
$$

Theorem 4. (Boundedness) Suppose $N_{k}=\left\{\left\{\alpha_{k} \mid P_{k}^{T_{k}}\right\},\left\{\beta_{k} \mid P^{I_{k}}\right\},\left\{\gamma_{k} \mid P_{k}^{F_{k}}\right\}\right\}$ indicate an PNHFE,

$$
\begin{aligned}
& N^{-}=\left\{\left\{\min \left\{\alpha_{k}\right\} \mid \min \left\{P_{k}^{T_{k}}\right\}\right\},\left\{\max \left\{\beta_{k}\right\} \mid \max \left\{P_{k}^{I_{k}}\right\}\right\},\left\{\max \left\{\gamma_{k}\right\} \mid \max \left\{P_{k}^{F_{k}}\right\}\right\}\right\}, \\
& N^{+}=\left\{\left\{\max \left\{\alpha_{k}\right\} \mid \max \left\{P_{k}^{T_{k}}\right\}\right\},\left\{\min \left\{\beta_{k}\right\} \mid \min \left\{P_{k}^{I_{k}}\right\}\right\},\left\{\min \left\{\gamma_{k}\right\} \mid \min \left\{P_{k}^{F_{k}}\right\}\right\}\right\} .
\end{aligned}
$$

Then,

$$
\operatorname{PNHFCOA}\left(N^{-}\right) \leq \operatorname{PNHFCOA}\left(N_{1}, N_{2}, \cdots, N_{n}\right) \leq \operatorname{PNHFCOA}\left(N^{+}\right) .
$$

Proof. $\forall N_{k}$, we know:

$$
\begin{aligned}
& \min \left\{\alpha_{k}\right\} \leq \alpha_{\pi(k)} \leq \max \left\{\alpha_{k}\right\}, \min \left\{\beta_{k}\right\} \leq \beta_{\pi(k)} \leq \max \left\{\beta_{k}\right\}, \min \left\{\gamma_{k}\right\} \leq \gamma_{\pi(k)} \leq \max \left\{\gamma_{k}\right\} \\
& \min \left\{P_{k}^{T_{k}}\right\} \leq P_{\pi(k)}^{T_{\pi(k)}} \leq \max \left\{P_{k}^{T_{k}}\right\}, \min \left\{P_{k}^{I_{k}}\right\} \leq P_{\pi(k)}^{I_{\pi(k)}} \leq \max \left\{P_{k}^{I_{k}}\right\}, \min \left\{P_{k}^{F_{k}}\right\} \leq P_{\pi(k)}^{F_{\pi(k)}} \leq \max \left\{P_{k}^{F_{k}}\right\} .
\end{aligned}
$$

Thus,

$$
\begin{aligned}
1-\prod\left(1-\alpha_{\pi(k)}\right)^{\left(\mu\left(F_{\pi(k)}\right)-\mu\left(F_{\pi(k-1)}\right)\right)} & \geq 1-\prod\left(1-\min \left\{\alpha_{i}\right\}\right)^{\left(\mu\left(F_{\pi(k)}\right)-\mu\left(F_{\pi(k-1)}\right)\right)} \\
& =1-\left(1-\min \left\{\alpha_{k}\right\}\right)^{\sum\left(\mu\left(F_{\pi(k)}\right)-\mu\left(F_{\pi(k-1)}\right)\right)} \\
& =\min \left\{\alpha_{k}\right\}=\min \left\{\alpha_{\pi(k)}\right\}, \\
\prod\left(\beta_{\pi_{(k)}}\right)\left(\mu\left(F_{\pi(k)}\right)-\mu\left(F_{\pi(k-1)}\right)\right) & \leq \prod\left(\max \left\{\beta_{k}\right\}\right)^{\left(\mu\left(F_{\pi(k)}\right)-\mu\left(F_{\pi(k-1)}\right)\right)} \\
& =\left(\max \left\{\beta_{k}\right\}\right)^{\sum\left(\mu\left(F_{\pi(k)}\right)-\mu\left(F_{\pi(k-1)}\right)\right)} \\
& =\max \left\{\beta_{k}\right\}=\max \left\{\beta_{\pi(k)}\right\},
\end{aligned}
$$




$$
\begin{aligned}
\prod\left(\gamma_{\pi(k)}\right)^{\left(\mu\left(F_{\pi(k)}\right)-\mu\left(F_{\pi(k-1)}\right)\right)} & \leq \prod\left(\max \left\{\gamma_{k}\right\}\right)^{\left(\mu\left(F_{\pi(k)}\right)-\mu\left(F_{\pi(k-1)}\right)\right)} \\
& =\left(\max \left\{\gamma_{k}\right\}\right)^{\sum\left(\mu\left(F_{\pi(k)}\right)-\mu\left(F_{\pi(k-1)}\right)\right)} \\
& =\max \left\{\gamma_{k}\right\}=\max \left\{\gamma_{\pi(k)}\right\} .
\end{aligned}
$$

For the probabilities, it is easy to get

$$
\begin{array}{r}
\prod \min \left\{P_{k}^{T_{k}}\right\}=\min \left\{P_{\pi(k)}^{T_{\pi(k)}}\right\} \leq \prod P_{\pi(k)}^{T_{\pi(k)}}, \\
\prod P_{\pi(k)}^{I_{\pi(k)}} \leq \prod \max \left\{P_{\pi(k)}^{I_{\pi(k)}}\right\}=\prod \max \left\{P_{i}^{I_{i}}\right\}, \\
\prod P_{\pi(k)}^{F_{\pi(k)}} \leq \prod \max \left\{P_{\pi(k)}^{F_{\pi(k)}}\right\}=\prod \max \left\{P_{i}^{F_{i}}\right\} .
\end{array}
$$

Therefore, we have:

$\operatorname{PNHFCOA}\left(N^{-}\right)=\bigcup\left\{\left\{\min \left\{\alpha_{\pi(k)}\right\} \mid \prod \min \left\{P_{k}^{T_{k}}\right\}\right\},\left\{\max \left\{\beta_{\pi(k)}\right\} \mid \prod \max \left\{P_{\pi(k)}^{I_{\pi(k)}}\right\}\right\},\left\{\max \left\{\gamma_{\pi(k)}\right\} \mid \prod \max \left\{P_{\pi(k)}^{F_{\pi(k)}}\right\}\right\}\right\}$.

By Definitions 7 and 9,

$$
\operatorname{PNHFCOA}\left(\mathrm{N}^{-}\right) \leq \operatorname{PNHFCOA}\left(N_{1}, N_{2}, \cdots, N_{n}\right) .
$$

Similarly, we can get

$$
\operatorname{PNHFCOA}\left(N_{1}, N_{2}, \cdots, N_{n}\right) \leq \operatorname{PNHFCOA}\left(N^{+}\right) .
$$

Theorem 5. (Idempotency) Supposing $\left.N_{i}=\left\{\left\{\alpha \mid P_{1}\right\},\left\{\beta \mid P_{2}\right\},\{\gamma] \mid P_{3}\right\}\right\}(i=1,2, \cdots, n)$ is a normalized PNHFE, $\mu$ is a fuzzy measure on $X$; then,

$$
\operatorname{PNHFCOA}\left(N_{1}, N_{2}, \cdots, N_{X}\right)=\left\{\left\{\alpha \mid P_{1}\right\},\left\{\beta \mid P_{2}\right\},\left\{\gamma \mid P_{3}\right\}\right\} .
$$

Proof. When $P_{1}, P_{2}, P_{3} \in\{1\}$, we have the following equation:

$$
\prod P_{j}=P_{j}(j=1,2,3) .
$$

Based on Definition 10, it is expressed by the following formula:

$$
\begin{aligned}
& \operatorname{PNHFCOA}\left(N_{1}, N_{2}, \cdots, N_{n}\right) \\
& =\left\{\left\{1-\prod(1-\alpha)^{\mu_{\pi(k)}} \mid \prod P_{1}\right\},\left\{\prod(\beta)^{\mu_{\pi(k)}} \mid \prod P_{2}\right\},\left\{\prod(\gamma)^{\mu_{\pi(k)}} \mid \prod P_{3}\right\}\right\} \\
& =\left\{\left\{1-(1-\alpha)^{\sum\left(\mu\left(F_{\pi(k)}\right)-\mu\left(F_{\pi(k)}\right)\right)} \mid P_{1}\right\},\left\{(\beta)^{\sum\left(\mu\left(F_{\pi(k)}\right)-\mu\left(F_{\pi(k)}\right)\right)} \mid P_{2}\right\},\left\{(\gamma)^{\sum\left(\mu\left(F_{\pi(k)}\right)-\mu\left(F_{\pi(k)}\right)\right)} \mid P_{3}\right\}\right\} \\
& =\left\{\left\{\alpha \mid P_{1}\right\},\left\{\beta \mid P_{2}\right\},\left\{\gamma \mid P_{3}\right\}\right\} .
\end{aligned}
$$

Theorem 6. (Commutativity) Suppose $A=\left\{N_{1}, N_{2}, \cdots, N_{n}\right\}$ and $B=\left\{N_{\lambda(1)}, N_{\lambda(2)}, \cdots, N_{\lambda(n)}\right\}$ are two finite sets. If the position of the element in $\left\{N_{\pi(1)}, N_{\pi(2)}, \cdots, N_{\pi(n)}\right\}$ is changed arbitrarily to get $\left\{N_{1}, N_{2}, \cdots, N_{n}\right\}$. Then,

$$
\operatorname{PNHFCOA}\left(N_{1}, N_{2}, \cdots, N_{n}\right)=\operatorname{PNHFCOA}\left\{N_{\lambda(1)}, N_{\lambda(2)}, \cdots, N_{\lambda(n)}\right\} .
$$

Proof. Based on Definition 10, the result is easy to get. 
Definition 11. Suppose $N_{k}=\left\{T_{k}\left|P^{T_{k}}, I_{k}\right| P^{I_{k}}, F_{k} \mid P^{F_{k}}\right\}$ indicates an PNHFE. Then, the PNHFCOG operator is described by the following formula:

$$
\begin{aligned}
& \operatorname{PNHFCOG}\left(N_{1}, N_{2}, \cdots, N_{n}\right)=\otimes_{\pi(k)=1}^{n} \mu_{\pi(k)} N_{\pi(k)} \\
& =\bigcup_{\alpha_{\pi(k)} \in T_{\pi(k)}, \beta_{\pi(k)} \in I_{\pi(k)}, \gamma_{\pi(k)} \in F_{\pi(k)}}\left\{\left\{\prod_{\pi(k)=1}^{n}\left(\alpha_{\pi(k)}\right)^{\mu_{\pi(k)}} \mid \prod_{\pi(k)=1}^{n} P_{\pi(k)}^{T_{\pi(k)}}\right\},\right. \\
& \left.\left\{1-\prod_{\pi(k)=1}^{n}\left(1-\beta_{\pi(k)}\right)^{\mu_{\pi(k)}} \mid \prod_{\pi(k)=1}^{n} P_{\pi(k)}^{I_{\pi(k)}}\right\},\left\{1-\prod_{\pi(k)=1}^{n}\left(1-\gamma_{\pi(k)}\right)^{\mu_{\pi(k)}} \mid \prod_{\pi(k)=1}^{n} P_{\pi(k)}^{F_{\pi}(k)}\right\}\right\},
\end{aligned}
$$

where $P_{\pi(k)}^{T_{\pi(k)}}, P_{\pi(k)}^{I_{\pi(k)}}$ and $P_{\pi(k)}^{F_{\pi(k)}}$ are the corresponding probabilities of $\alpha_{\pi(k)}, \beta_{\pi(k)}$ and $\gamma_{\pi(k)} \cdot \mu_{\pi(k)}=\mu\left(F_{\pi(k)}-\right.$ $\left.F_{\pi(k-1)}\right), F_{\pi(k)}=\left\{x_{\pi(1)}, x_{\pi(2)}, \cdots, x_{\pi(k)}\right\}$ and $F_{\pi(0)}=0$. The factor $n_{\pi(k)}$ hold $n_{\pi(1)} \geq n_{\pi(2)} \geq \cdots \geq$ $n_{\pi(n)}$

Theorem 7. Suppose that $N_{k}$ indicates an PNHFE, PNHFCOG $\left(N_{1}, N_{2}, \cdots, N_{n}\right)$ is still an PNHFE.

Similarly, the fuzzy measure satisfies different conditions, different types of PNHFCOA can be obtained.

(1) Assume $\mu(F)=1$, then

$$
\operatorname{PNHFCOG}\left(N_{1}, N_{2}, \cdots, N_{n}\right)=\max \left\{N_{1}, N_{2}, \cdots, N_{n}\right\} .
$$

(2) Assume $\mu(F)=0$, then

$$
\operatorname{PNHFCOG}\left(N_{1}, N_{2}, \cdots, N_{n}\right)=\min \left\{N_{1}, N_{2}, \cdots, N_{n}\right\} \text {. }
$$

(3) Assume the prerequisite $\mu\left(x_{\pi(k)}\right)=\mu\left(F_{\pi(k)}-F_{\pi}(i-1)\right)$ is independent, the PNHFCOG operator indicates an PNHFWG operator:

$$
\begin{aligned}
& \operatorname{PNHFWG}\left(N_{1}, N_{2}, \cdots, N_{n}\right)=\otimes_{k=1}^{n} \mu\left(x_{k}\right) N_{k} \\
& =\bigcup_{\alpha_{k} \in T_{k}, \beta_{k} \in I_{k}, \gamma_{k} \in F_{k}}\left\{\left\{\prod_{k=1}^{n}\left(\alpha_{k}\right)^{\mu\left(x_{k}\right)} \mid \prod_{k=1}^{n} P_{k}^{T_{k}}\right\},\left\{1-\prod_{k=1}^{n}\left(1-\beta_{k}\right)^{\mu\left(x_{k}\right)} \mid \prod_{k=1}^{n} P_{k}^{I_{k}}\right\},\left\{1-\prod_{k=1}^{n}\left(1-\gamma_{k}\right)^{\mu\left(x_{k}\right)} \mid \prod_{k=1}^{n} P_{k}^{F_{k}}\right\}\right\} .
\end{aligned}
$$

(4) Assume the precondition $\mu\left(x_{\pi(k)}\right)=\frac{1}{n}$, the PNHFFCG operator and PNHFWG operator reduce to the PNHFG operator:

$$
\begin{aligned}
& \operatorname{PNHFWG}\left(N_{1}, N_{2}, \cdots, N_{n}\right)=\otimes_{k=1}^{n} \mu\left(x_{k}\right) N_{k} \\
& =\bigcup_{\alpha_{k} \in T_{k}, \beta_{k} \in I_{k}, \gamma_{k} \in F_{k}}\left\{\left\{\prod_{k=1}^{n}\left(\alpha_{k}\right)^{\frac{1}{n}} \mid \prod_{k=1}^{n} P_{k}^{T_{k}}\right\},\left\{1-\prod_{k=1}^{n}\left(1-\beta_{k}\right)^{\frac{1}{n}} \mid \prod_{k=1}^{n} P_{k}^{I_{k}}\right\},\left\{1-\prod_{k=1}^{n}\left(1-\gamma_{k}\right)^{\frac{1}{n}} \mid \prod_{k=1}^{n} P_{k}^{F_{k}}\right\}\right\} .
\end{aligned}
$$

Theorem 8. By analyzing the PNHFCOA operator, we can obtain the following theorems, obviously.

(1) (Monotonicity) Assume $N_{k}=\left\{\left\{\alpha_{k} \mid P_{k}^{T_{k}}\right\},\left\{\beta_{k} \mid P_{k}^{I_{k}}\right\},\left\{\gamma_{k} \mid P_{k}^{F_{k}}\right\}\right\}$ and $\tilde{N}_{k}=\left\{\left\{\tilde{\alpha}_{k} \mid P_{k}^{\tilde{T}_{k}}\right\},\left\{\tilde{\beta}_{k} \mid P_{k}^{\tilde{I}_{k}}\right\}\right.$, $\left.\left\{\tilde{\gamma}_{k} \mid P_{k}^{\tilde{F}_{k}}\right\}\right\}$ indicate two PNHFEs. The factor $\pi(k)$ satisfies condition $N_{\pi(1)} \geq N_{\pi(2)} \geq \cdots \geq N_{\pi(n)}$ and $\tilde{N}_{\pi(1)} \geq \tilde{N}_{\pi(2)} \geq \cdots \geq \tilde{N}_{\pi(n)}$. With $\forall N_{\pi(k)}$ and $\forall \tilde{N}_{\pi(k)}$, there are $\alpha_{\pi(k)} \leq \tilde{\alpha}_{\pi(k)}, \beta_{\pi(k)} \geq \tilde{\beta}_{\pi(k)}$, $\gamma_{\pi(k)} \geq \tilde{\gamma}_{\pi(k)}$ and $P_{\pi(k)}^{T_{\pi(k)}}=P_{\pi(k)}^{\tilde{T}_{\pi(k)}}, P_{\pi(k)}^{I_{\pi(k)}}=P_{\pi(k)}^{\tilde{I}_{\pi(k)}}, P_{\pi(k)}^{F_{\pi(k)}}=P_{\pi(k)}^{\tilde{F}_{\pi(k)}}$. Then,

$$
\operatorname{PNHFCOG}\left\{N_{1}, N_{2}, \cdots, N_{n}\right\} \leq \operatorname{PNHFCOG}\left\{\tilde{N}_{1}, \tilde{N}_{2}, \cdots, \tilde{N}_{n}\right\} \text {. }
$$

(2) (Boundedness) Assume $N_{k}=\left\{\left\{\alpha_{k} \mid P_{k}^{T_{k}}\right\},\left\{\beta_{k} \mid P^{I_{k}}\right\},\left\{\gamma_{k} \mid P_{k}^{F_{k}}\right\}\right\}$ indicates an PNHFE, 


$$
\begin{aligned}
& N^{-}=\left\{\left\{\min \left\{\alpha_{k}\right\} \mid \min \left\{P_{k}^{T_{k}}\right\}\right\},\left\{\max \left\{\beta_{k}\right\} \mid \max \left\{P_{k}^{I_{k}}\right\}\right\},\left\{\max \left\{\gamma_{k}\right\} \mid \max \left\{P_{k}^{F_{k}}\right\}\right\}\right\}, \\
& N^{+}=\left\{\left\{\max \left\{\alpha_{k}\right\} \mid \max \left\{P_{k}^{T_{k}}\right\}\right\},\left\{\min \left\{\beta_{k}\right\} \mid \min \left\{P_{k}^{I_{k}}\right\}\right\},\left\{\min \left\{\gamma_{k}\right\} \mid \min \left\{P_{k}^{F_{k}}\right\}\right\}\right\} .
\end{aligned}
$$

Then,

$$
\operatorname{PNHFCOG}\left(N^{-}\right) \leq \operatorname{PNHFCOA}\left(N_{1}, N_{2}, \cdots, N_{n}\right) \leq \operatorname{PNHFCOG}\left(N^{+}\right)
$$

(3) (Idempotency) Assume $\left.N_{k}=\left\{\left\{\alpha \mid P_{1}\right\},\left\{\beta \mid P_{2}\right\},\{\gamma] \mid P_{3}\right\}\right\}$ is a normalized PNHFE, then

$$
\operatorname{PNHFCOG}\left(N_{1}, N_{2}, \cdots, N_{X}\right)=\left\{\left\{\alpha \mid P_{1}\right\},\left\{\beta \mid P_{2}\right\},\left\{\gamma \mid P_{3}\right\}\right\} \text {. }
$$

(4) (Commutativity) Assume $A=\left\{N_{1}, N_{2}, \cdots, N_{n}\right\}$ and $B=\left\{N_{\lambda(1)}, N_{\lambda(2)}, \cdots, N_{\lambda(n)}\right\}$ are two finite sets. If the position of the element in $\left\{N_{\pi(1)}, N_{\pi(2)}, \cdots, N_{\pi(n)}\right\}$ is changed arbitrarily to get $\left\{N_{1}, N_{2}, \cdots, N_{n}\right\}$, then:

$$
\operatorname{PNHFCOG}\left(N_{1}, N_{2}, \cdots, N_{n}\right)=\operatorname{PNHFCOG}\left\{N_{\lambda(1)}, N_{\lambda(2)}, \cdots, N_{\lambda(n)}\right\} .
$$

Lemma 1. By reference [37], if $x_{k} \geq 0, w_{k} \geq 0, \sum_{k=1}^{n}$, we have

$$
\prod_{k=1}^{n}\left(x_{k}\right)^{w_{k}} \leq \sum_{k=1}^{n} w_{k} x_{k}
$$

Theorem 9. Suppose $N_{k}=\left\{\left\{\alpha_{k} \mid P_{k}^{T_{k}}\right\},\left\{\beta_{k} \mid P_{k}^{I_{k}}\right\},\left\{\gamma_{k} \mid P_{k}^{F_{k}}\right\}\right\}$ indicates an PNHFE, we have

$$
\operatorname{PNHFCOG}\left(N_{1}, N_{2}, \ldots, N_{n}\right) \leq \operatorname{PNHFCOA}\left(N_{1}, N_{2}, \ldots, N_{n}\right) \text {. }
$$

Proof. Based on Lemma 1, Definitions 10 and 11, the following formula is obtained:

$$
\prod\left(\alpha_{\pi(k)}\right)^{\mu_{\pi(k)}} \leq \sum\left(\mu_{\pi(k)}\right) \alpha_{\pi(k)}=1-\sum \mu_{\pi(k)}\left(1-\alpha_{\pi(k)}\right) \leq 1-\prod\left(1-\alpha_{\pi(k)}\right)^{\mu_{\pi(k)}} .
$$

Obviously,

$$
\prod\left(\alpha_{\pi(k)}\right)^{\mu_{\pi(k)}} \prod P_{\pi(k)}^{T_{\pi(k)}} \leq\left(1-\prod\left(1-\alpha_{\pi(k)}\right)^{\mu_{\pi(k)}}\right) P_{\pi(k)}^{T_{\pi(k)}} .
$$

Similarly, we know

$$
\begin{aligned}
& \prod\left(\beta_{\pi(k)}\right)^{\mu_{\pi(k)}} \prod P_{\pi(k)}^{T_{\pi(k)}} \leq\left(1-\prod\left(1-\beta_{\pi(k)}\right)^{\mu_{\pi(k)}}\right) P_{\pi(k)}^{T_{\pi(k)}}, \\
& \prod\left(\gamma_{\pi(k)}\right)^{\mu_{\pi(k)}} \prod P_{\pi(k)}^{T_{\pi(k)}} \leq\left(1-\prod\left(1-\gamma_{\pi(k)}\right)^{\mu_{\pi(k)}}\right) P_{\pi(k)}^{T_{\pi(k)}} .
\end{aligned}
$$

Thus, based on Equation (4) and Definition 9,

$$
\operatorname{PNHFCOG}\left(N_{1}, N_{2}, \ldots, N_{n}\right) \leq \operatorname{PNHFCOA}\left(N_{1}, N_{2}, \ldots, N_{n}\right) .
$$

\section{A MADM method in PNHF Environment}

For a MAMD problem under the PNHF environment, assume $Z_{1}, Z_{2}, \cdots, Z_{n}$ indicates all the alternatives, $D_{1}, D_{2}, \cdots, D_{k}$ indicates all the attributes. The evaluated information of $Z_{i}$ with $D_{j}$ is indicated by PNHFE $N_{i j}=\left\{\left\{T_{i j} \mid P_{i j}^{T_{i j}}\right\},\left\{I_{i j} \mid P_{i j}^{I_{i j}}\right\},\left\{F_{i j} \mid P_{i j}^{F_{i j}}\right\}\right\}$.

Based on these necessary prerequisites, we elicit specific steps.

- $\quad$ Step 1. Construct a PNHF decision matrix (PNHFDM) $E=\left(N_{i j}\right)_{m \times k}$. 
Rank all PNHFEs from smallest to largest according to Definition 9. Then, the reorder decision matrix can be obtained such that $N_{i \pi(1)} \leq N_{i \pi(2)} \leq \cdots \leq N_{i \pi(n)},\{\pi(1), \pi(2), \cdots, \pi(n)\}$ indicates a new arrangement.

- $\quad$ Step 2. Calculate $\mu_{j}$ of criterion $D_{j}$.

In order to consider more interrelationships among criteria, by taking the $g_{\lambda}$ fuzzy measure, the measure $\mu$ of each criterion could be determined.

- $\quad$ Step 3. Based on the goal, select a PNHFCOA (PNHFCOG) operator to aggregate all PNHFEs $Z_{i}$ $(i=1,2, \cdots, n)$.

When we need to consider the group's major points, the PNHFCOA operator should be utilized. However, the individual major points could be emphasized based on the PNHFCOG operator. Thus, different types of operators can be used based on the different demands.

- $\quad$ Step 4. Reorder the alternatives

By the valued of $S\left(N_{i}\right), D\left(N_{i}\right)$ and Definition 9, all the $Z_{i}$ are ranked in decreasing order, DM picks an optimal option $Z_{i}$.

\section{The Program of the Proposed Approach}

Choosing the suitable 3PL plays a key role in business development, like improving efficiency and reducing costs, improving market share and service quality. ABC Machinery Manufacturing Company Limited as an automotive manufacturing company. The decision maker needs to select an optimal third part logistics supplier. There are four possible logistics suppliers that are denoted as $Z_{1}, Z_{2}, Z_{3}, Z_{4}$. The decision maker selects the following four attributes to access these alternatives: $D_{1}$ : equipment system; $D_{2}$ : effectiveness; $D_{3}$ : safety; $D_{4}$ : Correlation. The PNHFDM $E$ is obtained, depicted in Table 1. Suppose that fuzzy measures of $D_{j}$ are $\mu\left(D_{1}\right)=0.3, \mu\left(D_{2}\right)=0.3, \mu\left(D_{3}\right)=0.3$, $\mu\left(D_{4}\right)=0.2$, respectively.

- $\quad$ Step 1. Calculate the score values of all $Z_{i}$. The results are depicted in Table 2.

Thus, we can get reordered PNHFDM $E^{\prime}$. Because of space constraints, the decision matrix $E^{\prime}$ is omitted.

- Step 2. Since the information of fuzzy measure is $\mu\left(C_{1}\right)=0.3, \mu\left(C_{2}\right)=0.3, \mu\left(C_{3}\right)=0.3$, $\mu\left(C_{4}\right)=0.2$, respectively. By Equation (3), we get $\lambda=-0.2317$. Thus, taking $Z_{1}$ as an example, we can get

$$
\mu_{\pi(1)}=0.2477, \mu_{\pi(2)}=0.1732, \mu_{\pi(3)}=0.2791, \mu_{\pi(4)}=0.3 \text {. }
$$

- $\quad$ Step 3. Utilizing the PNHFCOA operator, by Equation (9), we can get

$$
S\left(Z_{1}\right)=0.6466, S\left(Z_{2}\right)=0.6436, S\left(Z_{3}\right)=0.5822, S\left(Z_{4}\right)=0.6950 .
$$

- Step 4. Rank the PNHFEs by Definition 9,

$$
Z_{4}>Z_{1}>Z_{2}>Z_{3}
$$

The 3PL Company $Z_{1}$ is an optimal option.

Next, suppose that the PNHFCOG operator is utilized to solve this problem. Similarly, the score value of alternative $A_{i}$ is obtained:

$$
S\left(Z_{1}\right)=0.6181, S\left(Z_{2}\right)=0.6167, S\left(Z_{3}\right)=0.5639, S\left(Z_{4}\right)=0.6686 .
$$


Thus, the final ranking of alternatives is determined, as follows:

$$
Z_{4}>Z_{1}>Z_{2}>Z_{3} .
$$

The 3PL Company $A_{1}$ is an optimal option.

Table 1. A PNHFDM Information E.

\begin{tabular}{|c|c|}
\hline & $C_{1}$ \\
\hline$A_{1}$ & $\begin{array}{l}\{\{0.5|0.3,0.57| 0.22,0.58|0.27,0.64| 0.21\},\{0.43|0.25,0.48| 0.2,0.49|0.30,0.55| 0.25\} \\
\{0.41|0.27,0.47| 0.23,0.52|0.23,0.46| 0.27\}\}\end{array}$ \\
\hline$A_{2}$ & $\begin{array}{l}\{\{0.44|0.27,0.49| 0.24,0.48|0.26,0.52| 0.23\},\{0.46|0.47,0.53| 0.53\} \\
\{0.29|0.18,0.33| 0.14,0.36|0.20,0.41| 0.16,0.41|0.18,0.47| 0.14\}\}\end{array}$ \\
\hline$A_{3}$ & $\begin{array}{l}\{\{0.41|0.30,0.48| 0.22,0.47|0.27,0.53| 0.21\},\{0.46|0.23,0.49| 0.26,0.49|0.24,0.53| 0.27\}, \\
\{0.39|0.24,0.41| 0.25,0.48|0.26,0.45| 0.25\}\}\end{array}$ \\
\hline \multirow[t]{2}{*}{$A_{4}$} & $\begin{array}{l}\{\{0.47|0.25,0.51| 0.24,0.50|0.26,0.53| 0.25\},\{0.34|0.33,0.43| 0.35,0.5 \mid 0.32\}, \\
\{0.42|0.28,0.45| 0.21,0.53|0.29,0.56| 0.22\}\}\end{array}$ \\
\hline & $C_{2}$ \\
\hline$A_{1}$ & $\begin{array}{l}\{\{0.40|0.26,0.51| 0.25,0.49|0.25,0.58| 0.24\},\{0.56|0.27,0.59| 0.24,0.60|0.26,0.63| 0.23\}, \\
\{0.39|0.23,0.43| 0.29,0.42|0.21,0.47| 0.27\}\}\end{array}$ \\
\hline$A_{2}$ & $\begin{array}{l}\{\{0.51|0.53,0.54| 0.47\},\{0.49|0.25,0.52| 0.22,0.57|0: 28,0.60| 0.25\}, \\
\{0.43|0.18,0.46| 0.18,0.48|0.17,0.50| 0.16,0.53|0.16,0.55| 0.15\}\}\end{array}$ \\
\hline$A_{3}$ & $\begin{array}{l}\{\{0.54|0.26,0.60| 0.25,0.63|0.25,0.68| 0.24\},\{0.50|0.48,0.56| 0.52\}, \\
\{0.43|0.26,0.46| 0.24,0.46|0.26 T, 0.50| 0.24\}\}\end{array}$ \\
\hline \multirow[t]{2}{*}{$A_{4}$} & $\begin{array}{l}\{\{0.61|0.54,0.67| 0.46\},\{0.43|0.27,0.50| 0.26,0.46|0.24,0.53| 0.23\}, \\
\{0.42|0.23,0.50| 0.24 T, 0.45|0.26,0.53| 0.27\}\}\end{array}$ \\
\hline & $C_{3}$ \\
\hline$A_{1}$ & $\begin{array}{l}\{\{0.56|0.24,0.62| 0.24,0.59|0.26,0.64| 0.26\},\{0.33|0.25,0.36| 0.24,0.37|0.26,0.41| 0.25\} \\
\{0.36|0.33,0.42| 0.36,0.45 \mid 0.31\}\}\end{array}$ \\
\hline$A_{2}$ & $\begin{array}{l}\{\{0.65|0.24 T, 0.69| 0.27,0.67|0.23,0.71| 0.26\},\{0.43|0.31,0.52| 0.23,0.46|0.27,0.55| 0.19\}, \\
\{0.43|0.26,0.46| 0.25,0.50|0.25,0.53| 0.24\}\}\end{array}$ \\
\hline$A_{3}$ & $\begin{array}{l}\{\{0.51|0.26,0.54| 0.26,0.57|0.24,0.60| 0.24\},\{0.43|0.26,0.46| 0.24,0.48|0.26,0.52| 0.24\} \\
\{0.49|0.25,0.54| 0.26,0.57|0.24,0.62| 0.25\}\}\end{array}$ \\
\hline \multirow[t]{2}{*}{$A_{4}$} & $\begin{array}{l}\{\{0.57|0.24,0.66| 0.28,0.66|0.22,0.73| 0.26\},\{0.43|0.54,0.49| 0.46\} \\
\{0.47|0.16,0.53| 0.17,0.56|0.16,0.50| 0.17,0.57|0.18,0.59| 0.17\}\}\end{array}$ \\
\hline & $C_{4}$ \\
\hline$A_{1}$ & $\begin{array}{l}\{\{0.48|0.47,0.57| 0.53\},\{0.40|0.51,0.47| 0.49\} \\
\{0.47|0.16,0.50| 0.15,0.53|0.15,0.49| 0.19,0.54|0.18,0.56| 0.17\}\}\end{array}$ \\
\hline$A_{2}$ & $\begin{array}{l}\{\{0.51|0.27,0.62| 0.26,0.54|0.24,0.64| 0.23\},\{0.40|0.25,0.46| 0.28,0.46|0.22,0.53| 0.25\}, \\
\{0.39|0.33,0.42| 0.37,0.45 \mid 0.30\}\}\end{array}$ \\
\hline$A_{3}$ & $\begin{array}{l}\{\{0.48|0.28,0.58| 0.23,0.51|0.26,0.61| 0: 23\},\{0.42|0.25,0.45| 0.24,0.47|0.26,0.50| 0.25\}, \\
\{0.42|0.27,0.50| 0.26,0.45|0.24,0.53| 0.23\}\}\end{array}$ \\
\hline$A_{4}$ & $\begin{array}{l}\{\{0.66|0.27,0.73| 0.24,0.71|0: 26,0.77| 0.23\},\{0.43|0.38,0.49| 0.33,0.54 \mid 0.29\}, \\
\{0.36|0.27,0.41| 0.24,0.39|0.26 T, 0.45| 0.23\}\}\end{array}$ \\
\hline
\end{tabular}

Table 2. The score values of PNHFE $N_{i j}$.

\begin{tabular}{ccccc}
\hline & $\boldsymbol{D}_{\mathbf{1}}$ & $\boldsymbol{D}_{\mathbf{2}}$ & $\boldsymbol{D}_{\mathbf{3}}$ & $\boldsymbol{D}_{\mathbf{4}}$ \\
\hline$Z_{1}$ & 0.6185 & 0.4700 & 0.8259 & 0.5782 \\
$Z_{2}$ & 0.6081 & 0.4885 & 0.7204 & 0.6941 \\
$Z_{3}$ & 0.5395 & 0.6181 & 0.5273 & 0.6072 \\
$Z_{4}$ & 0.5907 & 0.6825 & 0.6562 & 0.8329 \\
\hline
\end{tabular}

\section{Comparison with Other Approaches}

Based on the same problem background, the comparison results are described. 
Wu's method emphasizes the individual (group's) major points, which utilize the MADM problems with a simplified neutrosophic environment.

Peng put forward the TOPSIS-based QUALIFIEX method and the cross-entropy measurement to manage MADM situations with probability multi-valued neutrosophic elements. Then, the effectiveness of this method is demonstrated by an illustrative example.

However, in many actual problems, attributes are not independent. But by comparing the above methods, the association between attributes is not considered. However, attribute correlation is considered in our proposed method. The decision results are more reasonable and effective. The final results by different approaches are indicated in Table 3.

Table 3. Choice of optimal result.

\begin{tabular}{cccc}
\hline Method & Sort of Results & Optimal Alternative & Worst Alternative \\
\hline TOPSIS-based QUALIFLEX method [3] & $Z_{4}>Z_{2}>Z_{1}>Z_{3}$ & $Z_{4}$ & $Z_{3}$ \\
SNNPWA operator [45] & $Z_{3}>Z_{1}>Z_{2}>Z_{4}$ & $Z_{3}$ & $Z_{4}$ \\
SNNPWG operator [45] & $Z_{3}>Z_{2}>Z_{1}>Z_{4}$ & $Z_{2}$ & $Z_{1}$ \\
PNHFCOA operator & $Z_{4}>Z_{1}>Z_{2}>Z_{3}$ & $Z_{4}$ & $Z_{3}$ \\
PNHFCOG operator & $Z_{4}>Z_{1}>Z_{2}>Z_{3}$. & $Z_{4}$ & $Z_{3}$ \\
\hline
\end{tabular}

Through the above analysis, the DMs' evaluation information is represented by PNHFEs. PNHFEs are more flexible in describing the hesitant MADM information and reporting the probabilistic values of all hesitant values. The Choquet integral and aggregation operators are integrated in our method. Next, the alternatives' information is aggregated and ranked. In this model, the interrelationship between attributes are involved by Choquet integral. The MADM problems are effectively resolved by utilizing interdependencies or interactions between attributes. The results are closer to the actual situations.

\section{Conclusions}

Firstly, our goal is to aggregate the PNHF information by the notion of PNHFS. By applying the Choquet integral, the weight information is extended, more information about the correlation between attributes is mined. The advantage of PNHFS is that it can explain the preferences of DMs without information loss. By investigating, we found both of the PNHFCOA and PNHFCOG operators satisfying the following characteristics: monotonity, boundedness, idempotency and commutativity. Those operators can reduce to some common aggregation operators. Moreover, those aggregation operators were used to an PNHF background, where fuzzy measure of attributes are recognized. All alternatives are reordered and choose an optimal option. Next, we present an illustrative situation to explain the objectivity of our method. The result received by the PNHFCOA and PNHFCOG operators are effective and flexible. The results are more suitable for the actual situations, because more information can be considered based on our method. Thus, when some uncertain problems or inconsistent and indeterminate information needs to be resolved, our proposed approach shows great advantages. In regard to the next jobs, more types of aggregation operators are investigated and applied in other practical situations, like medical diagnoses, group decision-making, risk evaluations, and fractal-wavelet modeling [46-50].

Author Contributions: All authors have contributed equally to this paper. The idea of the this paper is described by X.Z. The framework of the paper is constructed and analyzed by S.S. and Q.Z.

Funding: This research was funded by the National Natural Science Foundation of China (Grant No. 61573240).

Conflicts of Interest: The authors declare no conflict of interest. 


\section{References}

1. Konwar, N.; Davvaz, B.; Debnath, P. Results on generalized intuitionistic fuzzy hypergroupoids. J. Intell. Fuzzy Syst. 2019, 36, 2571-2580. [CrossRef]

2. Fu, C.; Chang, W.; Xue, M.; Yang, S. Multiple criteria group decision-making with belief distributions and distributed preference relations. Eur. J. Oper. Res. 2019, 273, 623-633. [CrossRef]

3. Peng, H.G.; Zhang, H.Y.; Wang, J.Q. Probability multi-valued neutrosophic sets and its application in multi-criteria group decision-making problems. Neural Comput. Appl. 2017, 20, 563-583. [CrossRef]

4. Xue, J.; Wu, C.; Chen, Z.; Van Gelder, P.H.; Yan, X. Modeling human-like decision-making for inbound smart ships based on fuzzy decision trees. Expert Syst. Appl. 2019, 115, 172-188. [CrossRef]

5. Spizzichino, F.L. On the probabilistic meaning of copula-based extensions of fuzzy measures. Applications to target-based utilities and multi-state reliability systems. Fuzzy Sets Syst. 2019, 354, 1-19. [CrossRef]

6. Ma Y.C.; Zhang, X.H.; Yang, X.F.; Zhou X. Generalized neutrosophic extended triplet group. Symmetry 2019, 11, 327. [CrossRef]

7. Li, L.; Jin, Q.; Hu, K. Lattice-valued convergence associated with CNS spaces. Fuzzy Sets Syst. 2018. [CrossRef]

8. Jin, Q.; Li, L.; Lv, Y.; Zhao, F.; Zou, J Connectedness for lattice-valued subsets in lattice-valued convergence spaces. Quaest. Math. 2019, 42, 135-150. [CrossRef]

9. Li, L.Q. p-Topologicalness-A Relative Topologicalness in T-Convergence Spaces. Mathematics 2019, 7, 228. [CrossRef]

10. Zhang, X.H.; Park, C.; Wu, S.P. Soft set theoretical approach to pseudo-BCI algebras. J. Intell. Fuzzy Syst. 2018, 34, 559-568. [CrossRef]

11. Wu, X.Y.; Zhang, X.H. The decomposition theorems of AG-neutrosophic extended triplet loops and strong AG-(1, 1)-loops. Mathematics 2019, 7, 268. [CrossRef]

12. Zhang, X.H. Fuzzy anti-grouped filters and fuzzy normal filters in pseudo-BCI algebras. J. Intell. Fuzzy Syst. 2017, 33, 1767-1774. [CrossRef]

13. Brito, V.T.F.; Ferreira, F.A.F.; Pérez-Gladish, B.; Govindan, K.; et.al. Developing a green city assessment system using cognitive maps and the Choquet integral. J. Clean. Prod. 2019, 218, 486-497. [CrossRef]

14. Krishnan, A.; Wahab, S.; Kasim, M.; Bakar, E. An alternate method to determine $\lambda^{0}$-measure values prior to applying Choquet integral in a multi-attribute decision-making environment. Decis. Sci. Lett. 2019, 8, $193-210$. [CrossRef]

15. Beg I.; Jamil R N.; Rashid T. Diminishing Choquet Hesitant 2-Tuple Linguistic Aggregation Operator for Multiple Attributes Group Decision Making. Int. J. Anal. Appl. 2019, 17, 76-104.

16. Zadeh, L.A. Fuzzy sets. Inf. Control. 1965, 8, 338-353. [CrossRef]

17. Castillo, O.; Atanassov, K. Comments on Fuzzy Sets, Interval Type-2 Fuzzy Sets, General Type-2 Fuzzy Sets and Intuitionistic Fuzzy Sets. In Recent Advances in Intuitionistic Fuzzy Logic Systems; Springer: Cham, Switzerland, 2019; pp. 35-43.

18. Song, C.; Zhao, H.; Xu, Z.S.; Hao, Z. Interval probabilistic hesitant fuzzy set and its application in the Arctic geopolitical risk evaluation. Int. J. Intell. Syst. 2019, 34, 627-651. [CrossRef]

19. Farhadinia, B.; Xu, Z.S. Distance Measures for Hesitant Fuzzy Sets and Their Extensions; Springer: Singapore, 2019; pp. 31-58.

20. Smarandache, F. A unifying field in logics: Neutrosophic logic. Multi-Valued Log. 1999, 8, 489-503.

21. Wei, G.; Zhang, Z. Some single-valued neutrosophic Bonferroni power aggregation operators in multiple attribute decision-making. J. Ambient Intell. Humaniz. Comput. 2019, 10, 863-882. [CrossRef]

22. Thong, N.T.; Dat, L.Q.; Hoa, N.D.; Ali, M.; Smarandache, F. Dynamic interval valued neutrosophic set: Modeling decision-making in dynamic environments. Comput. Ind. 2019, 108, 45-52. [CrossRef]

23. Sun, R.; Hu, J.; Chen, X. Novel single-valued neutrosophic decision-making approaches based on prospect theory and their applications in physician selection. Soft Comput. 2019, 23, 211-225. [CrossRef]

24. Yong, R.; Ye, J. Multiple Attribute Projection Methods with Neutrosophic Sets. In Fuzzy Multi-Criteria Decision-Making Using Neutrosophic Sets; Springer: Cham, Switzerland, 2019; pp. 603-622.

25. Ye, J.; Du, S. Some distances, similarity and entropy measures for interval-valued neutrosophic sets and their relationship. Int. J. Mach. Learn. Cybern. 2019, 10, 347-355. [CrossRef]

26. Sahin, R.; Liu, P.D. Maximizing deviation method for neutrosophic multiple attribute decision-making with incomplete weight information. Neural Comput. Appl. 2015, 27, 2017-2029. [CrossRef] 
27. Ye, J. Improved cosine similarity measures of simplified neutrosophic sets for medical diagnoses. Artif. Intell. Med. 2015, 63, 171-179. [CrossRef]

28. Tian, Z.P.; Zhang, H.Y.; Wang, J. Wang, J.Q.; Chen, X.H. Multi-criteria decision-making method based on a cross-entropy with interval neutrosophic sets. Int. J. Syst. Sci. 2016, 47, 3598-3608. [CrossRef]

29. Zhang, X.H.; Borzooei, R.A. and Jun, Y.B. Q-filters of quantum B-algebras and basic implication algebras. Symmetry. 2018, 10, 573. [CrossRef]

30. Zhang, X.H.; Wang, X.J.; Smarandache, F.; Jaíyéolá, T.G.; Lian, T. Singular neutrosophic extended triplet groups and generalized groups. Cogn. Syst. Res. 2018. [CrossRef]

31. Zhang, X.H.; Bo, C.X.; Smarandache, F.; Park, C. New operations of totally dependent-neutrosophic sets and totally dependent-neutrosophic soft sets. Symmetry 2018, 10, 187. [CrossRef]

32. Zhang, X.H.; Mao, X.Y.; Wu, Y.T.; Zhai, X.H. Neutrosophic filters in pseudo-BCI algebras. Int. J. Uncertainty Quant. 2018, 8, 511-526. [CrossRef]

33. Smith, P. Exploring public transport sustainability with neutrosophic logic. Transp. Plan. Technol. 2019, 1-17. [CrossRef]

34. Biswas, P.; Pramanik, S.; Giri, B.C. Neutrosophic TOPSIS with group decision-making. In Fuzzy Multi-Criteria Decision-Making Using Neutrosophic Sets; Springer: Cham, Switzerland, 2019; pp. 543-585.

35. Nirmal, N.P.; Bhatt, M.G. Development of Fuzzy-Single Valued Neutrosophic MADM Technique to Improve Performance in Manufacturing and Supply Chain Functions. In Fuzzy Multi-Criteria Decision-Making Using Neutrosophic Sets; Springer, Cham, Switzerland, 2019; pp. 711-729.

36. Li, G.; Niu, C.; Zhang, C. Multi-criteria decision-making approach using the fuzzy measures for environmental improvement under neutrosophic environment. Ekoloji 2019, 28, 1605-1615.

37. Shao, S.T.; Zhang, X.H.; Li, Yu.; Bo, C.X. Probabilistic single-valued (interval) neutrosophic hesitant fuzzy set and its application in multi-attribute decision-making Symmetry 2018, 10, 419. [CrossRef]

38. Choquet, G. Theory of capacities. Ann. Inst. Fourier 1953, 5, 131-295. [CrossRef]

39. Khan, M.S.A.; Abdullah, S.; Ali, A.; Amin, F.; Hussain, F. Pythagorean hesitant fuzzy Choquet integral aggregation operators and their application to multi-attribute decision-making. Soft Comput. 2019, 23, 251-267. [CrossRef]

40. Corrente, S.; Greco, S.; Slowiński, R. Robust ranking of universities evaluated by hierarchical and interacting criteria. In Multiple Criteria Decision Making and Aiding; Springer: Cham, Switzerland, 2019; pp. 145-192.

41. Labreuche, C.; Grabisch, M. Using multiple reference levels in Multi-Criteria Decision aid: The Generalized-Additive Independence model and the Choquet integral approaches. Eur. J. Oper. Res. 2018, 267, 598-611. [CrossRef]

42. Yager, R.R. On Using the Shapley Value to Approximate the Choquet Integral in Cases of Uncertain Arguments. IEEE Trans. Fuzzy Syst. 2018, 26, 1303-1310. [CrossRef]

43. Liu, P.; Tang, G. Some generalized Shapely interval-valued dual hesitant fuzzy uncertain linguistic Choquet geometric operators and their application to multiple attribute decision-making. J. Intell. Fuzzy Syst. 2019, 36, 557-574. [CrossRef]

44. Sugeno, M. Theory of Fuzzy Integrals and Its Applications. Ph.D. Thesis, Tokyo Institute of Technology, Tokyo, Japan, 1974.

45. Wu, X.H.; Wang, J.; Peng, J.J.; Chen, X.H. Cross-entropy and prioritized aggregation operator with simplified neutrosophic setsand their application in multi-criteria decision-making problems.Int. J. Fuzzy Syst. 2016, 18, 1104-1116. [CrossRef]

46. Guariglia, E. Primality, Fractality and Image Analysis. Entropy 2019, 21, 304. [CrossRef]

47. Guido, R.C.; Addison, P.; Walker, J. Introducing wavelets and time-frequency analysis. IEEE Eng. Biol. Med. Mag. 2009, 28, 13. [CrossRef]

48. Guido, R.C. Practical and useful tips on discrete wavelet transforms. IEEE Signal Process. Mag. 2015, 32, C162-C166 . [CrossRef]

49. Guariglia, E. Entropy and Fractal Antennas. Entropy 2016, 18, 84. [CrossRef]

50. Guariglia, E. Harmonic Sierpinski Gasket and Applications. Entropy 2018, 20, 714. [CrossRef]

(c) 2019 by the authors. Licensee MDPI, Basel, Switzerland. This article is an open access article distributed under the terms and conditions of the Creative Commons Attribution (CC BY) license (http:/ / creativecommons.org/licenses/by/4.0/). 